\title{
Time-resolved photoelectron spectroscopy of organic molecules in aqueous solutions.
}

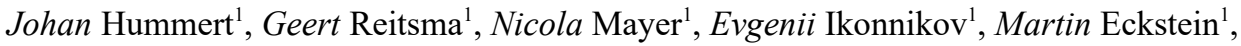 \\ and Oleg Kornilov ${ }^{1,1}$ \\ ${ }^{1}$ Max Born Institute for Nonlinear Optics and Short Pulse Spectroscopy, Max-Born-Straße 2A, 12489 \\ Berlin, Germany
}

\begin{abstract}
We use time-resolved photoelectron spectroscopy with wavelength-selected XUV femtosecond pulses to study photoinduced dynamics of organic molecules in solutions at millimolar concentrations. Upon electronic excitation we observe relaxation processes, such as excited state intramolecular proton transfer and trans-cis isomerization.
\end{abstract}

\section{INTRODUCTION}

Photoelectron spectroscopy is a powerful analytical tool which brought many insights into the electronic structure of molecules, clusters and solids. Combined with femtosecond and attosecond sources of extreme ultraviolet (XUV) laser radiation it offers the opportunity to directly follow transient electronic structure of excited states on unprecedented timescales. However, collecting photoelectron spectra from volatile liquid samples has proven to be a difficult task because of the high vapor pressure of most common solvents, such as water or ethanol. Nonetheless liquid phase spectroscopy is deemed especially important in investigation of molecular dynamics, since many molecules of interest, such as biochromophores or molecular switches, cannot be delivered in gas phase at sufficient densities and, more importantly, their functionality often depends on the solvent environment.

In 1973 Kai and Hans Siegbahn were the first to introduce photoelectron spectroscopy for liquid samples [1]. However, the method received a wide attention later in 1997, when M. Faubel, B. Steiner and J. P. Toennies introduced the liquid jet technology delivering samples into a vacuum system as liquid microjets tens of micrometers in diameter [2]. Since then numerous static photoelectron spectroscopy studies were performed at synchrotrons [3]. XUV time-dependent photoelectron spectroscopy also has been developed for liquid jets, however it has been limited to studies of pure solvents or samples with very high solubilities $[4,5,6]$. In this contribution we extend these studies to dilute millimolar samples of organic molecules in aqueous solutions.

1 Corresponding author: kornilov@mbi-berlin.de 

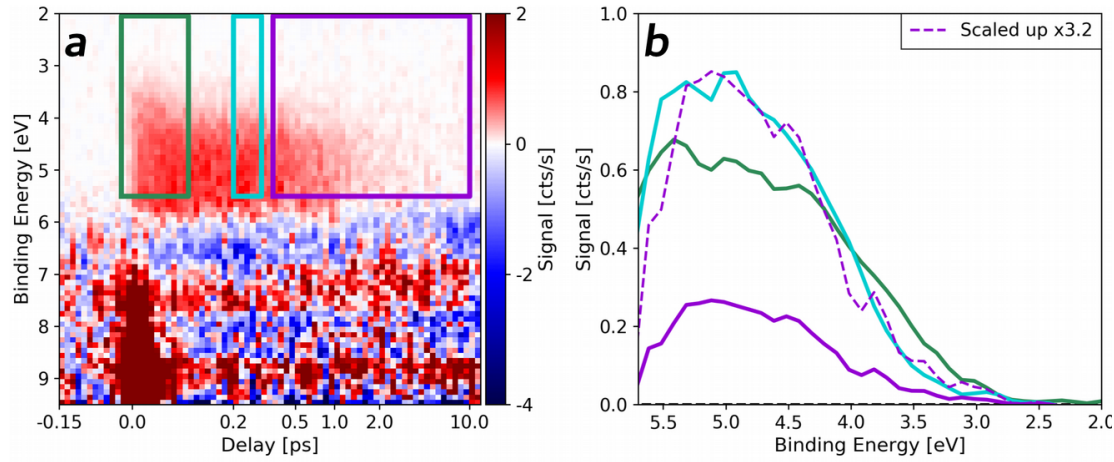

Figure 1: a) False color 2D difference map (the signal at negative delays is used for subtraction) of time-resolved photoelectron spectra of Methyl Orange in aqueous solution. Red color corresponds to signals enhanced by the $400 \mathrm{~nm}$ pump pulse, while blue colors correspond to depletion. The strong enhancement of the signal around zero time corresponds to liqht-assisted photoionization of water molecules, while the weaker band at low binding energies (3-6 eV) reflects electronic relaxation of the molecule. b) Averaged transient photoelectron spectra extracted from the map in part a) (see colored boxes). At early time delays the spectrum shifts to higher binding energies, while at later delays the excited state signals decay without further spectral changes.

\section{RESULTS AND DISCUSSION}

We employ femtosecond XUV pulses generated by high-order harmonic generation (HHG). The pulses are filtered by a time-delay-compensating monochromator beamline employing conical diffraction gratings. The beamline was recently used in our studies of dynamics of highly excited nitrogen cations [5] and neutral nitrogen molecules [6] revealing major dissociation pathways in the former case and an intriguing stabilization phenomenon for overlapping autoionizing resonances in the latter. In this contribution we combine this beamline with a liquid microjet and a "magnetic bottle" time-of-flight spectrometer. A pump-probe configuration with $400 \mathrm{~nm}$ pump pulses and XUV probe pulses allows us to detect light-assisted photoelectric effect at zero time overlap [7].

When organic yellow dye molecules are added to water we observe an additional feature in XUV-only photoelectron spectra corresponding to ionization of the several molecular orbitals. We find that in a large range of solubilities the magnitude of the signal detected in photoelectron spectra is negatively correlated to the solubility of the molecule. This result emphasizes the surface sensitivity of the photoelectron spectroscopy in liquid samples.

In the time-resolved experiments we investigate dynamics in three organic yellow dye molecules: Methyl Orange, Metanil Yellow and Quinoline Yellow. The experimental results for Methyl Orange are shown in Figure 1. The molecules are excited by $400 \mathrm{~nm}$ pulses and are probed by XUV pulses with photon energy of $26.5 \mathrm{eV}$ (spectral bandwidth of about 0.5 $\mathrm{eV}$ ). A time resolution of $45 \mathrm{fs}$ could be achieved in our setup. Figure 1a) presents spectra for a range of pump-probe time delays as a false color map. The spectra for negative time delays (XUV pulse arrives before the $400 \mathrm{~nm}$ pulse) are used for subtraction to emphasize the difference induced by the pump pulse. Clearly visible is a new photoelectron band appearing at zero time delay in the range of 3 to $6 \mathrm{eV}$ binding energies. This contribution corresponds to Methyl Orange molecules excited by the $400 \mathrm{~nm}$ photons. In the first hundred femtosecond the binding energy in this band increases, while at longer time delays 
the spectrum of the band remains constant and only its intensity decreases with a timescale of about 1 ps. This is best visible in Figure 1b), where averaged spectra are shown for three ranges of time delays.

We observe ultrafast stabilization of the excited state energy for all three molecules investigated in the present experiments. This fast change of the photoelectron spectrum is attributed to the solvent cage rearrangement, which is followed by electronic relaxation on one or several timescales specific to the solute. For the case of Quinoline Yellow the decay is reminiscent of the recently proposed excited state intramolecular proton transfer [10]. The relaxation of Methyl Orange and Metanil Yellow is consistent with ultrafast trans-cis isomerization typical for azo-benzene derivatives.

\section{CONCLUSIONS}

In this contribution we present the first experiments on XUV time-resolved photoelectron spectroscopy of dilute solutions of organic molecules at millimolar concentrations. We combine a recently developed time-delay-compensating monochromator beamline with a liquid microjet and an efficient time-of-flight spectrometer and follow relaxation dynamics of several organic molecules excited by visible femtosecond laser pulses. The observed relaxation timescales and changes of transient photoelectron spectra suggest ultrafast solvent rearrangement plays a role at short times. At longer times (few picoseconds), relaxation involves conformational changes, such as trans-cis isomerization in azo-benzene derivatives.

We acknowledge the funding of this project by Deutsche Forschungsgemeinschaft (grant KO 4920/11). The authors are grateful to Kathrin Aziz-Lange and Reinhard Großer for their work on the construction of the experiment endstation and to Boris Peev, Katrin Herrmann and Roman Peslin for their support during the experimental campaign.

\section{References}

[1] H. Siegbahn and K. Siegbahn, J. El. Spec. and Rel. Phenom. 2, 319 (1973)

[2] M. Faubel, B. Steiner, and J. P. Toennies, J. Chem. Phys. 106, 9013 (1997)

[3] R. Seidel, B. Winter, and S. E. Bradforth, Annu. Rev. Phys. Chem. 67, 283 (2016)

[4] K. R. Siefermann, Y. Liu, E. Lugovoy, O. Link, M. Faubel, U. Buck, B. Winter, and B. Abel, Nat. Chem. 2, 274 (2010)

[5] N. Engel, S. I. Bokarev, A. Moguilevski, A. A. Raheem, R. Al-Obaidi, T. Möhle, G.

Grell, K. R. Siefermann, B. Abel, S. G. Aziz, O. Kuhn, M. Borgwardt, I. Y. Kiyan, and E. F. Aziz, Phy. Chem. Chem. Phys. 19, 14248 (2017)

[6] J. Ojeda et al, Phys. Chem. Chem. Phys. 19, 17052 (2017)

[7] M. Eckstein, C.-H. Yang, M. Kubin, F. Frassetto, L. Poletto, H.-H. Ritze, M. J. J.

Vrakking, and O. Kornilov, J. Phys. Chem. Lett. 6, 419 (2015)

[8] M. Eckstein, C.-H. Yang, F. Frassetto, L. Poletto, G. Sansone, M. J. J. Vrakking, and O.

Kornilov, Phys. Rev. Lett. 116, 163003 (2016)

[9] C. Arrell, J. Ojeda, L. Mewes, J. Grilj, F. Frassetto, L. Poletto, F. van Mourik, and M.

Chergui, Phys. Rev. Lett. 117, 143001 (2016)

[10] G. R. Han et al, Sci. Reports 7, 10.1038/s41598-017-04114-9 (2017) 